\title{
Utilisation d'eau à basse température pour le forçage de cultures sous abris. II. Effets du chauffage du sol sur la croissance et le développement de plantes issues de bulbes (glaïeul, iris, tulipe, lis)
}

\author{
Thierry BOULARD \& Alain BAILLE \\ avec la collaboration technique de J. LORGEOU \& G. SAPPE \\ I.N.R.A., Station de Bioclimatologie, Centre de Recherches d'Avignon, F 84140 Montfavet.
}

RÉSUMÉ

\begin{abstract}
Le chauffage du sol par l'intermédiaire de tuyaux enterrés modifie profondément les champs de température dans la zone racinaire des plantes. Pour des espèces comme l'iris, la tulipe et le glaïeul, le chauffage se traduit par une accélération de la croissance et du développement des plantes, tant que les futurs organes aériens sont situés sous la surface du sol. Par contre, pour le lis, dont l'apex reste très peu de temps sous la surface du sol, l'effet du chauffage du sol est pratiquement nul ; l'influence du chauffage du sol est donc directement liée aux caractéristiques de croissance des plantes.

Sur le plan de la qualité des plantes, on observe un antagonisme entre la qualité et la précocité.
\end{abstract}

Mots clés additionnels : Chauffage du sol, forçage de bulbes.

Using warm water for forcing greenhouse crops. II. Effects of soil warming on growth and development of bulbous flowers (gladiolus, iris, tulip, lily).

Soil heating by means of buried pipes greatly modifies the temperature range in the root zone of plants. In the case of iris, tulip and gladiolus, soil heating leads to an acceleration of growth and development of the young plants, as long as the top of the stem remains below the soil surface. Soil heating has practically no effect on lily, whose stem stays only a short time below the soil surface. The influence of soil heating depends mainly on the growth characteristics of the plant. An opposition between quality and earliness has been observed.

Additional key words : Soil warming, forcing bulbs.

\section{INTRODUCTION}

L'utilisation d'eau tiède $\left(20\right.$ à $\left.40^{\circ} \mathrm{C}\right)$ pour le chauffage des serres nécessite l'emploi d'échangeurs de chaleur de grande surface, localisés dans le sol ou près du sol. Ces systèmes modifient sensiblement le champ de température dans la zone racinaire des plantes (BOULARD \& BAILLE, 1984).

L'influence d'une élévation de la température des racines sur le comportement de cultures sous abris a été l'objet de nombreuses études, menées principalement sur des plantes maraîchères et, en tout premier lieu, sur la tomate : CORnillon (1974), COOPER \& ThORnley (1976). Cependant, pour un certain nombre d'espèces comme les plantes à bulbe, l'élévation de la température du sol ne concerne pas uniquement leurs racines, mais intéresse également le bulbe lui même et se traduit le plus souvent par une accélération de la croissance et du développement des organes aériens de la plante qui est bien supérieure à celle que l'on peut observer pour d'autres espèces (BERNINGER, 1977 ; BOULARD et al., 1981).

$\mathrm{Ce}$ comportement particulier des plantes à bulbes en sol chauffé a été mis en évidence par les essais de FORTANIER \& ZEVENBERGEN (1973) sur iris. BERNINGER (1977, 1978) a étendu les études à un éventail plus large de plantes bulbeuses (tulipe, iris, glaïeul) et a montré que l'accélération de la croissance et du développement des plantes sur sol chauffé était très importante pour toutes ces espèces.

Compte tenu de ces éléments, des essais de chauffage pour la production de fleurs coupées issues de bulbes (tulipe, iris, glaïeul, lis) ont été programmés sur le site expérimental de St-Laurent-des-Eaux (BoulARD \& BAILLE, 1984), afin de micux appréhender les effets d'un chauffage de sol à partir d'eaux de rejet sur ce type de plantes. 


\section{MÉTHODES D'ÉTUDE ET DISPOSITIFS EXPÉRI- MENTAUX}

\section{A. Spécificité du matériel végétal utilisé}

On sait depuis les travaux de BlaAuw (1941) et HaRTSEMA (1961) que l'évolution du bulbe d'iris et de tulipe et du cormus de glaieul est très influencée par l'origine du bulbe et par le traitemert thermique qu'il a pu recevoir pendant sa phase de repos.

Très schématiquement, ces travaux ont mis en évidence que, dans un premier temps, des températures élevées permettent au bulbe d'acquérir l'aptitude à différencier une fleur; puis, le passage à température basse ou moyenne permet l'élongation ultérieure de la fleur et de la tige. Les températures de conservation du bulbe conditionnent done le degré de différenciation du bourgeon floral au moment de la plantation pour la tulipe et l'iris et, dans une moindre mesure, pour le glaïeul, et vont avoir une action sur le développement ultérieur de la plante.

Les travaux de LE NARD (1980) effectués sur la tulipe et l'iris ont mis en évidence l'influence que la températurc de conservation du bulbe exerçait également sur les processus de mobilisation des réserves du bulbe et de bulbification. Il est donc délicat d'interpréter des différences de croissance et de développement qui portent sur des bulbes de différentes origines ou dont les durées de conservation sont différentes.

\section{B. Dispositif expérimental}

En fonction de ces observations, nous avons donc ćtć amenés à comparer, dans nos essais, des bulbes de même origine, conservés dans les mêmes conditions (ou ayant subi des traitements thermiques identiques) et plantés aux mêmes dates puis soumis à des équilibres de températures air/sol différents. Sur ce plan, il convient de distinguer:

- les essais effectués sous 2 tunnels plastique où, pour sensiblement les mêmes températures d'air (tabl. 1), il a ćté maintenu dans l'un d'eux, une température du sol (à $-0,1 \mathrm{~m}$ ) d'environ 4 à $5^{\circ} \mathrm{C}$ plus élevée ;

- les essais en plein champ, qui concernent les glaieuls, pour lesquels nous avons pu maintenir 4 niveaux différents de température du sol par l'utilisation seule ou croisée des techniques de chauffage du sol et de couverture plastique du sol: sol témoin nu, sol témoin couvert, sol chauffé nu, sol chauffé couvert (tabl. 2).

\section{Disposition des essais, matériel végétal}

Dans la mesure du possible, nous avons mis en place des cultures dans des conditions proches de celles de la production, tant au niveau du traitement thermique des bulbes, des densités de plantation, des cultivars qu'au niveau des tailles des parcelles élémentaires qui sont au minimum de $2 \mathrm{~m}^{2}$ pour les tulipes, iris, lis et de $4 \mathrm{~m}^{2}$ pour les glaieuls. Les tableaux $3 a$ et $3 b$ récapitulent la liste des principaux essais mis en place, les cultivars et les préparations thermiques utilisées sous abris (lis, iris, tulipe, glaïcul) et en plein champ (glaïcul).

\section{Suivi des températures}

Il a été réalisé par l'intermédiairc de sondes à résistance de platine :

- dans des prises ventilées à $+50 \mathrm{~cm}$ pour la température d'air,

- à $-10 \mathrm{~cm}$ de profondeur pour la température du sol.

\section{TABLEAU 1}

Températures moyennes de sol et d'air $\left({ }^{\circ} \mathrm{C}\right)$ mesurées de novembre à mars dans le tunnel chauffé par l'air et par le sol et dans le témoin chauffé uniquement par l'air. (Campagnes 79-80 et 80-81.) Average air and soil temperature (from November to March) in the air-soil heated tunnel and in the control.

\begin{tabular}{|c|c|c|c|c|}
\hline & \multicolumn{2}{|c|}{$\begin{array}{c}\text { Tunnel chauffé } \\
\text { /air }+ \text { sol }\end{array}$} & \multicolumn{2}{|c|}{$\begin{array}{c}\text { Tunnel chauffé } \\
\text { /air }\end{array}$} \\
\hline & $\begin{array}{l}\text { T air } \\
(0,5 \mathrm{~m})\end{array}$ & $\begin{array}{c}\text { T sol } \\
(-0,1 \mathrm{~m})\end{array}$ & $\begin{array}{l}\mathrm{T} \text { air } \\
(0,5 \mathrm{~m})\end{array}$ & $\begin{array}{c}\text { T sol } \\
(-0,1 \mathrm{~m})\end{array}$ \\
\hline Campagne $79-80$ & 11,5 & 14,7 & 10,9 & 9,8 \\
\hline Campagne $80-81$ & 11 & 14,2 & 11,2 & 9,8 \\
\hline
\end{tabular}

\section{TABLEAU 2}

Températures moyennes de sol et d'air $\left({ }^{\circ} \mathrm{C}\right)$ mesurées de mars à mai 1981 dans les cultures de glaieuls plein champ menées avec différentes techniques de forçage.

Average air and soil temperature (from March to May 1981) of gladiolus crops in the open, with soil heating and mulching.

\begin{tabular}{lcc}
\hline \hline \multicolumn{1}{c}{ Traitements } & T sol $(-0,1 \mathrm{~m})$ & T air $(+0,5 \mathrm{~m})$ \\
\hline Sol chauffé couvert & 18,9 & 13,9 \\
\hline Sol chauffé nu & 17,4 & 12 \\
\hline Sol non chauffé couvert & 14,9 & 13,3 \\
\hline Sol non chauffé nu & 13,0 & 12,0 \\
\hline
\end{tabular}

L'ensemble des sondes était relié à une acquisition de données sur cassettes qui intégrait les mesures sur un pas de temps horaire.

\section{Suivi des paramètres de croissance et des stades de développement}

\section{Croissance}

Des prélèvements destructifs de 12 individus au minimum par répétition ont été opérés une fois par semaine. On a mesuré systématiquement la taille des différents organes aériens (feuilles, tiges, entrenœuds, bouton floral) et leur position par rapport à la surface du sol ainsi que le poids frais et sec de la partie aérienne, du bulbe-mère et des bulbes-fils.

\section{Développement}

Deux stades de développement ont été suivis: la floraison (observations journalières), la différenciation florale (observation à la loupe binoculaire) soit au moment de la plantation du bulbe (iris, tulipe, lis), soit en cours de végétation sur des plantes prélevées 2 fois par semaine (glaiicul). Pour cette dernière espèce, la comparaison du nombre de fleurs différenciées à une date donnée par rapport au nombre de fleurons observés à la floraison permet de déterminer le stade de développement où, en moyenne, 90 p. 100 des fleurons sont différenciés. 
TABLEAU $3 a$

Réduction de la durée de la phase plantation-floraison sur sol chauffé pour les différentes cultivars de glä̈euls plantés sous tunnels et en plein champ $\left({ }^{*}\right)$.

Reduction in duration of the "planting-50\% flowering " phase on heated soil for different cultivars of gladiolus in tunnels and in the open (*).

\begin{tabular}{|c|c|c|c|c|c|}
\hline $\begin{array}{c}\text { Cultivars } \\
\text { préparation thermique }\end{array}$ & $\begin{array}{l}\text { Date de } \\
\text { plantation }\end{array}$ & $\begin{array}{l}\text { Différence moyenne } \\
\text { de température entre } \\
\text { sol chauffé et témoin } \\
\left({ }^{\circ} \mathrm{C}\right)\end{array}$ & $\begin{array}{l}\text { Durée de la phase } \\
\text { plantation-floraison } \\
\text { en témoin } \\
\text { (jours) }\end{array}$ & $\begin{array}{r}\text { Réd } \\
\text { durée } \\
\text { planta } \\
\text { sur } \\
\text { (jours) }\end{array}$ & $\begin{array}{l}\text { la } \\
\text { lase } \\
\text { ison } \\
\text { fé } \\
(\%)\end{array}$ \\
\hline \multicolumn{6}{|l|}{ Glä̈euls } \\
\hline 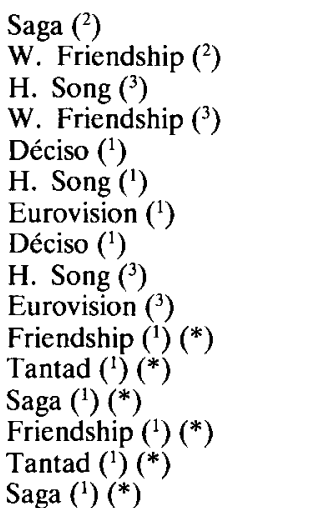 & $\begin{array}{l}24 / 1 / 80 \\
24 / 1 / 80 \\
14 / 2 / 80 \\
14 / 2 / 80 \\
26 / 1 / 81 \\
26 / 1 / 81 \\
26 / 1 / 81 \\
16 / 2 / 81 \\
16 / 2 / 81 \\
16 / 2 / 81 \\
17 / 3 / 80 \\
17 / 3 / 80 \\
17 / 3 / 80 \\
17 / 3 / 80 \\
17 / 3 / 80 \\
17 / 3 / 80\end{array}$ & $\begin{array}{l}4 \\
4 \\
4 \\
4 \\
4,4 \\
4,4 \\
4,4 \\
4 \\
4 \\
4 \\
4 \\
4 \\
4 \\
4,4 \\
4,4 \\
4,4\end{array}$ & $\begin{array}{l}126 \\
124 \\
110 \\
113 \\
126 \\
115 \\
137 \\
118 \\
110 \\
118 \\
121 \\
115 \\
119 \\
132 \\
126 \\
128\end{array}$ & $\begin{array}{l}17 \\
17 \\
18 \\
15 \\
16 \\
15 \\
18 \\
12 \\
13 \\
12 \\
14 \\
15 \\
19 \\
18 \\
19 \\
18\end{array}$ & $\begin{array}{l}13 \\
14 \\
20 \\
13 \\
13 \\
13 \\
13 \\
10 \\
12 \\
10 \\
12 \\
13 \\
16 \\
14 \\
15 \\
14\end{array}$ \\
\hline
\end{tabular}

Préparations thermiques des bulbes : ( $\left.{ }^{1}\right)$ Non préparés ; $\left({ }^{2}\right) 4$ semaines à $20^{\circ} \mathrm{C} ;\left({ }^{3}\right) 6$ semaines à $20^{\circ} \mathrm{C}$.

TABLEAU $3 b$

Réduction de la durée de la phase plantation-floraison sur sol chauffé pour les différentes espèces et cultivars plantés sous tunnels.

Reduction in duration of the "planting-50\% flowering " phase on heated soil for different cultivars of iris, tulip and lily in tunnels.

\begin{tabular}{ccccc}
\hline \hline $\begin{array}{c}\text { Cultivars } \\
\text { préparation thermique }\end{array}$ & $\begin{array}{c}\text { Différence moyenne } \\
\text { plantation }\end{array}$ & $\begin{array}{c}\text { de température entre } \\
\text { sol chauffé et témoin }\end{array}$ & $\begin{array}{c}\text { Durée de la phase } \\
\text { plantation-floraison } \\
\text { en témoin } \\
\text { (jours) }\end{array}$ & $\begin{array}{c}\text { Réduction de la } \\
\text { durée de la phase } \\
\text { plantation-floraison } \\
\text { sur sol chauffé } \\
(\%)\end{array}$ \\
(jours) & $\left({ }^{\circ} \mathrm{C}\right)$ & & $(\%)$
\end{tabular}

Iris

Ideal ( $\left(^{1}\right)$

W. Wedgwood ( $\left.{ }^{1}\right)$

Ideal $\left(^{1}\right)$

Prof. Blaauw $\left({ }^{2}\right)$

W. Wedgwood $\left({ }^{3}\right)$

Ideal $\left(^{3}\right)$

Prof. Blaauw $\left({ }^{4}\right)$

Tulipes

G. Apeldoorn $\left({ }^{5}\right)$
Apeldoorn $\left({ }^{6}\right)$
E. Peeck $\left(^{6}\right)$
P. Richter $\left(^{6}\right)$
Gander $\left(^{6}\right)$
K. Nelis $\left(^{6}\right)$

K. Nelis $\left.{ }^{6}\right)$

Lis

Enchantement $\left({ }^{7}\right)$

Enchantement $\left({ }^{8}\right)$
W. Wedgwood (1)
$8 / 11 / 79$

$8 / 11 / 79$

$12 / 12 / 79$

$9 / 01 / 80$

$9 / 01 / 80$

$21 / 10 / 80$

$21 / 10 / 80$

$5 / 12 / 80$

$21 / 10 / 80$

$5 / 12 / 80$

$8 / 12 / 80$

$8 / 12 / 80$

$5 / 12 / 80$

$5 / 12 / 80$

$21 / 10 / 80$

$3 / 02 / 81$
4,5

4,5

5

4,5

4,5

4,4

4,4

4,4

4,4

4,4

4,4

4,4

4,4

4,4

4,4

5,5
112

118

98
107

97

115

111

$\begin{array}{lll}85 & 16 & 19 \\ 84 & 16 & 19 \\ 71 & 11 & 15 \\ 79 & 13 & 16 \\ 78 & 14 & 18 \\ 82 & 12 & 15\end{array}$

136

100

18

$\begin{array}{ll}19 & 14 \\ 23 & 19 \\ 20 & 17 \\ 15 & 15 \\ 16 & 15 \\ 18 & 18 \\ 20 & 17 \\ 19 & 17\end{array}$

$$
19
$$

19

16

Préparations thermiques: $\left({ }^{1}\right) 30^{\circ} \mathrm{C}+6$ semaines à $9{ }^{\circ} \mathrm{C}+2$ semaines à $17^{\circ} \mathrm{C} ;\left({ }^{2}\right) 30^{\circ} \mathrm{C}+8$ semaines à $9{ }^{\circ} \mathrm{C}+2$ semaines à $17^{\circ} \mathrm{C}$; $\left(^{3}\right) 30{ }^{\circ} \mathrm{C}+9$ semaines à $13{ }^{\circ} \mathrm{C} ;\left({ }^{4}\right) 30^{\circ} \mathrm{C}+11$ semaines à $13{ }^{\circ} \mathrm{C} ;\left({ }^{5}\right) 20^{\circ} \mathrm{C}+12$ semaines à $5{ }^{\circ} \mathrm{C} ;\left({ }^{6}\right) 20^{\circ} \mathrm{C}+9$ semaines à $5^{\circ} \mathrm{C} ;\left({ }^{7}\right)$ lis retardés; $\left(^{8}\right)$ lis non retardés. 
III. INFLUENCE DE LA TEMPERATURE DU SOL SUR LA CROISSANCE ET LE DÉVELOPPEMENT DES FLEURS COUPÉES ISSUES DE BULBES

En se reportarit aux tableaux $3 a$ et $3 b$ qui récapitulent les gains de précocité obtenus pour une élévation de la température du sol de 4 à $5^{\circ} \mathrm{C}$, on constate que l'iris, la tulipe et le glaïeul voient leur durée totale de culture réduite sensiblement du même pourcentage: 10 à $20 \mathrm{p}$. 100 , soit 2 à 3 semaines. Ce résultat montre d'ores et déjà un premier intérêt du chauffage du sol pour toutes ces espèces: le raccourcissement de la période de chauffage.

Le lis se différencie des autres espèces, le chauffage du sol ne s'accompagnant que d'un gain de précocité très faible pour cette espèce. Ces résultats confirment, dans l'ensemble, les observations faites aux Pays-Bas sur l'iris, soit en phytotron (FORTANIER \& ZEVENBERGEN, 1973) avec des températures de sol et d'air assez élevées $\left(15^{\circ} \mathrm{C}\right.$ en air, $18^{\circ} \mathrm{C}$ en sol) et un faible niveau d'éclairement, soit sous serre (SCHIPPER \& VAN DIJK, 1979) également avec des températures de sol et d'air assez fortes $\left(15\right.$ à $21^{\circ} \mathrm{C}$ en air et en sol). Cette différence de niveau de température explique sans doute que l'on n'ait pas observé, comme ces auteurs, d'avortements de boutons floraux lorsque le niveau d'éclairement est faible et pour les floraisons de décembre à janvier. Avec sensiblement les mêmes températures de sol et d'air que celles des essais de FORTANIER \& ZEVENBERGEN, mais sous serre et dans une région ensoleillée (à Fréjus, en région méditerranéenne), BERNINGER (1977) ne signale pas d'avortement des fleurs mais remarque, pour l'iris, la tulipe et le glaïeul, une perte de qualité (principalement une perte de poids des plantes).

On retrouve là l'opposition entre la réduction de la durée du cycle de développement des plantes et la qualité du produit obtenu, opposition que l'on a pu observer dans la plupart de nos essais et qui pose le problème de l'effet de la température du sol sur la cinétique de croissance et de développement en fonction du niveau d'éclairement.

\section{A. Effet sur la croissance}

Pour les 4 especes étudiées (lis, tulipe, iris, gläieul), on observe que la croissance de la partie aérienne est accéléréc en sol chauffé presque uniquement en début de culture. L'accroissement de la température du sol accélère l'enracinement et stimule la croissance des organes aériens et des bulbes-fils. La figure 1 montre l'évolution de la longueur de la tige de l'iris, de la plantation à la floraison, en sols chauffé et non chauffé. On remarque que l'accélération de la croissance en sol chauffé se produit en début de culture ; une évolution analogue est observée pour le poids frais de la partie aérienne et des bulbes-fils de tulipe (fig. 2). Pour souligner la liaison entre la température du sol et l'accélération de la croissance, nous avons représenté sur la figure 3 l'évolution de la taille de la tige du glaïeul, avec 4 niveaux différents de température du sol. On peut observer une accélération de la croissance tant que la tige se trouve tout entière sous la surface du sol. Ces observations laissent donc supposer que la température du sol intervient directement sur la croissance en s'appliquant aux zones d'ćlongation de la future partie aérienne de la plante.

Si l'influence directe de la température du sol sur l'ensemble des zones en croissance en début de culture a pu être facilement rnise en évidence, il est par contre plus difficile d'analyser l'influence de la température du sol sur chaque organe pris séparément (bulbe, racine, tige, feuille).

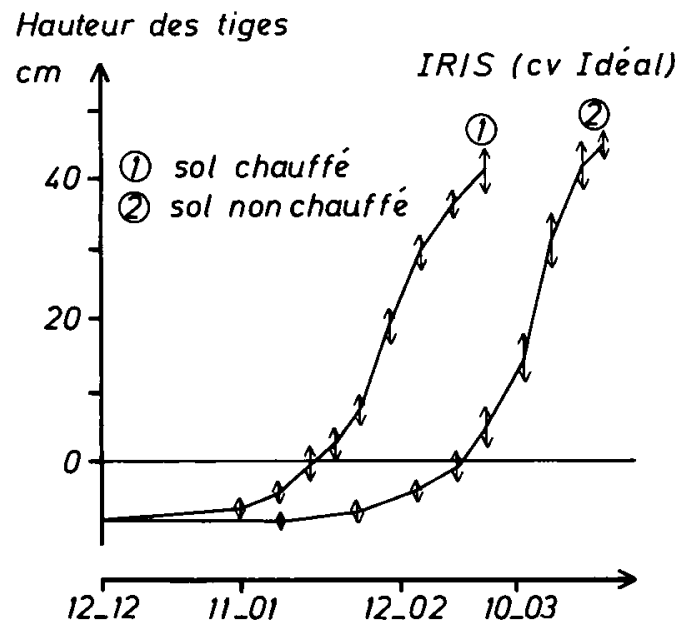

Figure 1

Evolution de la longueur de la tige de l'iris en sol chauffé $\left(\simeq 15^{\circ} \mathrm{C}\right)$ et non chauffé $\left(\simeq 10^{\circ} \mathrm{C}\right)$.

Increase in stem length in iris with heated soil $\left(15^{\circ} \mathrm{C}\right)$ and non-heated soil $\left(10^{\circ} \mathrm{C}\right)$ conditions.

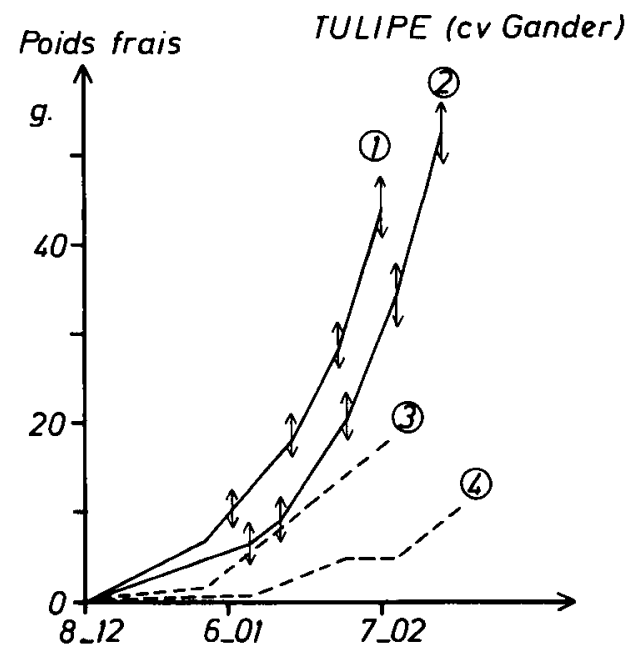

Figure 2

Evolution du poids frais:

- de la partie aérienne de la plante de tulipe (cv Gander, plantation du 5/12/80): (1) en sol chauffé, (2) en sol non chauffé,

- et des bulbes-fils: (3) en sol chauffé, (4) en sol non chauffé. Change in fresh weight of:

- aerial parts of tulips (cv Gander, planted 12/05/80) : (1) heated soil, (2) non-heated soil,

- daughter bulbs: (3) heated soil, (4) non-heated soil.

\section{B. Effet sur le développement}

L'étude de l'effet de la température du sol sur le développement a été menée principalement par le biais de l'analyse de la durée des différentes phases de développement.

On a pu analyser, pour toutes les espèces, la durée de la phase plantation-floraison (tabl. $3 a$ et $3 b$ ) et déterminer ainsi la réduction de cette phase due au chauffage.

Dans le cas du glaỉeul, qui est la seule espèce dont la fleur n'est pas encore différenciée au moment de la plantation, nous pouvons situer cette avance de développement dès le stade de différenciation de l'épi floral, c'est-à-dire à un stade beaucoup plus précoce que le stade de floraison. Dans lc cas des glaieuls de plein champ menés avec 4 niveaux 


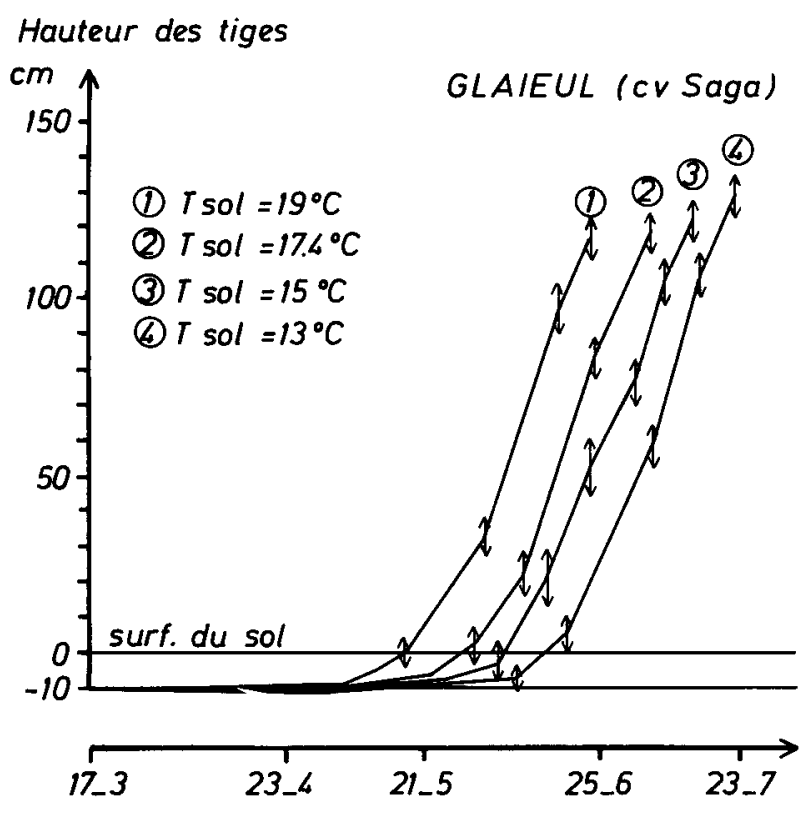

Figure 3

Evolution de la hauteur des tiges de glaïeuls en plein champ avec différentes températures de sol (obtenues par l'utilisation du chauffage du sol et du paillage).

Increase in the height of gladiolus stems in open air crops with different soil temperature conditions (obtained with soil heating and mulching).

différents de température de sol, on a représenté (fig. 4) la durée des phases de développement «plantation - 90 p. 100 de fleurons différenciés" et "plantation-floraison».

Comme lors de l'analyse du phénomène de croissance, on observe que l'essentiel de l'avance de développement est acquis à un stade précoce, lorsque les ébauches florales et les fleurs sont encore situées sous la surface du sol. Ce phénomène est général: si on met en relation l'avance observée à la floraison et la durée pendant laquelle la fleur (ou les ébauches florales) est située sous la surface du sol, on constate (fig. 5) que ces 2 grandeurs sont directement proportionnelles pour les 4 espèces testées.

Afin de préciser le mode d'action de la température du sol sur le développement, nous avons utilisé la méthode de la somme de températures sur la phase de développement qui va de la plantation à la floraison (DURAND, 1969 ; DURAND et al., 1982). Cette méthode part du principe que, déduction faite d'un seuil de développement qui est spécifique de chaque espèce (et même de chaque cultivar), la somme de température que la plante a reçue (exprimée en degré-jour) est une valeur constante pour une phase de développement donnée. Sur la phase de développement plantation-floraison, elle a été utilisée pour l'iris par FORTANIER \& ZEVENBERGEN (1973) ainsi que, pour le glaïeul et la tulipe, par BERNINGER (1977). A partir de ces essais, les seuils de développement suivants ont été calculés: $5^{\circ} \mathrm{C}$ pour le glaieul, $3^{\circ} \mathrm{C}$ pour la tulipe, $4{ }^{\circ} \mathrm{C}$ pour l'iris et le lis.

En reprenant ces valeurs, on a appliqué cette méthode aux données obtenues dans nos essais.

$\mathrm{Si}$ on prend comme exemple les glaïeuls en plein champ, on constate (tabl. 4) que les sommes de températures calculées sur la phase plantation-floraison à partir de la température de sol ou à partir de la température d'air présentent des écarts importants entre traitements, supérieurs à $100^{\circ} \mathrm{C}$ jours. Par contre, si on effectue le calcul des sommes de température en utilisant successivement :

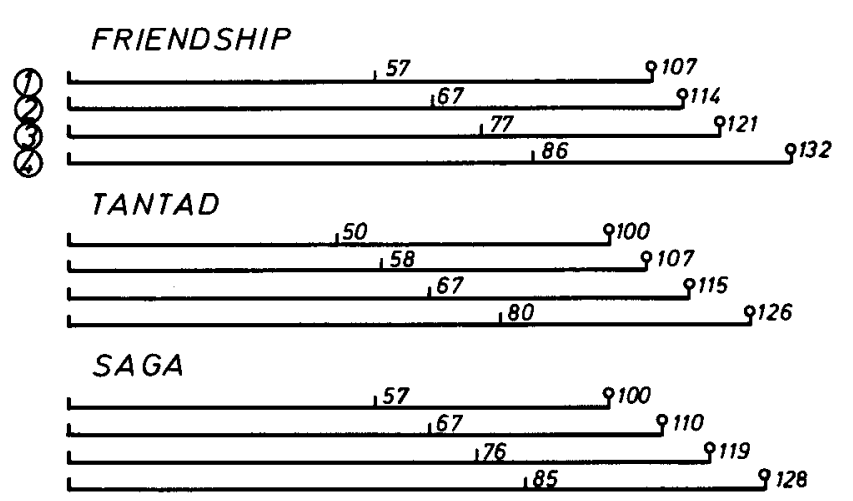

1 Fin de la différentiation florale

P Floraison $50 \%$

$$
\begin{aligned}
& \text { (1) } T \text { sol }=19^{\circ} \mathrm{C} \\
& \text { (2) } T \text { sol }=17.4^{\circ} \mathrm{C} \\
& \text { (3) } T \text { sol }=15^{\circ} \mathrm{C} \\
& \text { (4) } T \text { sol }=13^{\circ} \mathrm{C}
\end{aligned}
$$

Figure 4

Glaïeuls plein champ : variation de la durée des phases de développement :

- "plantation-différenciation florale " $(90 \%$ des fleurons différenciés),

- "plantation-floraison" (50\% de floraison) en fonction de la température du sol.

Open air gladiolus crop : variation in the duration of the phases of development :

- "plantation-flower initiation" (90\% florets initiated),

- "plantation-flowering" (50\% flowering) as a function of soil temperature.

Gain de précocité

en sol chauffe

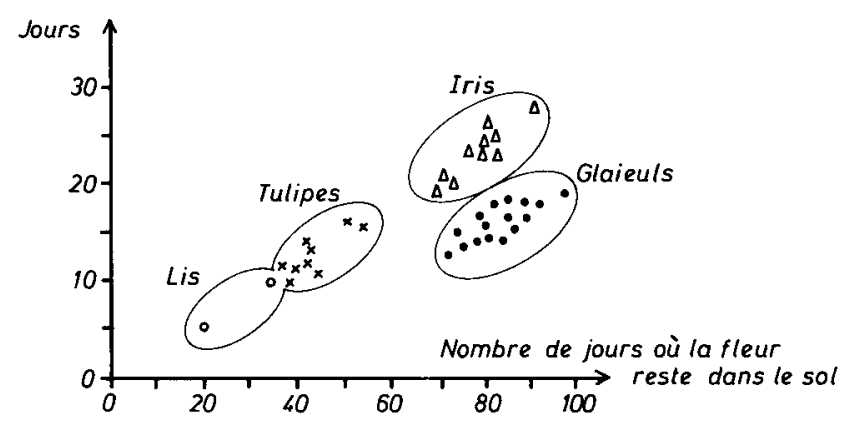

Figure 5

Efficacité du chauffage du sol sur le raccourcissement de la durée de culture en fonction de la durée pendant laquelle la fleur et l'apex demeurent dans la surface du sol.

Relation between precocity of flowering and duration of the period for which the bud and the flower stay under the soil surface.

- la température de sol tant que la fleur n'a pas passé la surface dh sol,

- la température d'air ensuite,

on observe que les différences dans les sommes de température sont beaucoup plus faibles $\left(20\right.$ à $30^{\circ} \mathrm{C}$-jour $)$ et pratiquement constantes pour une variété donnée.

On obtient les mêmes résultats pour les autres espèces ; l'exemple de l'iris est donné tableau 5: les écarts entre sommes de température sont beaucoup plus faibles lorsque les sommes sont calculées d'abord avec la température de sol et ensuite avec la température d'air. 
TABLEAU 4

Valeurs des sommes de température calculées de la plantation à la floraison du glaïeul (seuil de développement $5^{\circ} \mathrm{C}$ ) pour 4 équilibres de température air-sol, avec différentes méthodes de prise en compte du champ de température en fonction du stade de la plante.

Heat sum values for gladiolus (base temperature $: 5^{\circ} \mathrm{C}$ ) with different soil temperatures and different methods for taking into account the range of temperatures according to the stage of the plants.

\begin{tabular}{|c|c|c|c|c|c|}
\hline \multirow[b]{2}{*}{ Glaieuls } & \multicolumn{5}{|c|}{ Sommes de degré-jours $\left({ }^{\circ} \mathrm{C} \cdot\right.$ jours $)$} \\
\hline & $\begin{array}{l}\text { Sol chauffé } \\
\text { couvert } \\
\text { T sol } \simeq 19^{\circ}\end{array}$ & $\begin{array}{l}\text { Sol chauffé } \\
\text { nu } \\
\text { T sol } \simeq 17,4^{\circ}\end{array}$ & $\begin{array}{l}\text { Sol témoin } \\
\text { couvert } \\
\mathrm{T} \text { sol } \simeq 15^{\circ}\end{array}$ & $\begin{array}{l}\text { Sol témoin } \\
\text { nu } \\
\mathrm{T} \text { sol } \simeq 13^{\circ}\end{array}$ & $\begin{array}{l}\text { Ecart } \\
\text { moyen }\end{array}$ \\
\hline $\begin{array}{ll} & \left({ }^{1}\right) \\
& \\
& \left({ }^{2}\right)\end{array}$ & $\begin{array}{r}1139 \\
1474 \\
959\end{array}$ & $\begin{array}{r}1079 \\
1424 \\
846\end{array}$ & $\begin{array}{l}1122 \\
1253 \\
1066\end{array}$ & $\begin{array}{l}1124 \\
1148 \\
1087\end{array}$ & $\begin{array}{r}22 \\
151 \\
111\end{array}$ \\
\hline cv Tantad & $\begin{array}{r}1063 \\
1385 \\
890\end{array}$ & $\begin{array}{r}996 \\
1331 \\
775\end{array}$ & $\begin{array}{l}1060 \\
1180 \\
1002\end{array}$ & $\begin{array}{r}1032 \\
1090 \\
991\end{array}$ & $\begin{array}{r}17 \\
135 \\
105\end{array}$ \\
\hline $\begin{array}{l}(1) \\
\left({ }^{2}\right) \\
\left({ }^{3}\right)\end{array}$ & $\begin{array}{r}1072 \\
1395 \\
900\end{array}$ & $\begin{array}{r}1029 \\
1379 \\
814\end{array}$ & $\begin{array}{l}1066 \\
1229 \\
1044\end{array}$ & $\begin{array}{l}1057 \\
1115 \\
1013\end{array}$ & $\begin{array}{r}16 \\
133 \\
106\end{array}$ \\
\hline
\end{tabular}

(1) Prise en compte de $\mathrm{T}$ sol, puis de $\mathrm{T}$ air en fonction de la position de la fleur ; $\left({ }^{2}\right)$ Prise en compte uniquement de $\mathrm{T}$ sol $(-10 \mathrm{~cm})$; $\left({ }^{3}\right)$ Prise en compte uniquement de $\mathrm{T}$ air $(+50 \mathrm{~cm})$.

\section{TABLEAU 5}

Valeurs des sommes de température calculées de la plantation à la floraison de l'iris (seuil de développement $4{ }^{\circ} \mathrm{C}$ ) pour 2 équilibres de température air-sol, avec différentes méthodes de prise en compte du champ de température en fonction du stade de la plante.

Heat sum values for iris (base temperature : $4^{\circ} \mathrm{C}$ ) on heated and nonheated soil with different methods for taking into account the temperaure range and the stages of the plants.

\begin{tabular}{|c|c|c|c|}
\hline & \multicolumn{3}{|c|}{ Sommes de degré-jour $\left({ }^{\circ} \mathrm{C} \cdot\right.$ jours $)$} \\
\hline Iris & $\begin{array}{l}\text { Tunnel } \\
\text { air }+ \text { sol } \\
\text { chauffé }\end{array}$ & $\begin{array}{l}\text { Tunnel } \\
\text { air } \\
\text { chauffé }\end{array}$ & Ecart \\
\hline $\begin{array}{ll} & \left({ }^{1}\right) \\
\text { cv W. Wedgwood } & \left(^{2}\right) \\
\text { plant. nov. } 79 & \left({ }^{3}\right)\end{array}$ & $\begin{array}{l}645 \\
584 \\
888\end{array}$ & $\begin{array}{l}642 \\
670 \\
535\end{array}$ & $\begin{array}{l}+\quad 3 \\
-\quad 86 \\
+353\end{array}$ \\
\hline $\begin{array}{ll} & \left({ }^{1}\right) \\
\text { cv W. Wedgwood } & \left(^{2}\right) \\
\text { plant. déc. } 79 & \left(^{3}\right)\end{array}$ & $\begin{array}{l}694 \\
595 \\
870\end{array}$ & $\begin{array}{l}707 \\
763 \\
639\end{array}$ & $\begin{array}{r}-13 \\
-168 \\
+231\end{array}$ \\
\hline $\begin{array}{ll} & \left(^{1}\right) \\
\text { cy W. Wedgwood } & \left(^{2}\right) \\
\text { plant. janv. } 80 & \left(^{3}\right)\end{array}$ & $\begin{array}{l}667 \\
596 \\
816\end{array}$ & $\begin{array}{l}669 \\
709 \\
611\end{array}$ & $\begin{array}{l}-2 \\
-113 \\
+205\end{array}$ \\
\hline
\end{tabular}

(1) Prise en cornpte de $\mathrm{T}$ sol, puis de $\mathrm{T}$ air en fonction de la position de la fleur; $\left({ }^{2}\right)$ Prise en compte uniquement de $\mathrm{T}$ sol $(-10 \mathrm{~cm}) ;\left({ }^{3}\right)$ Prise en compte uniquement de $\mathrm{T}$ air $(+50 \mathrm{~cm})$.

Pour bien comprendre le mode d'action de l'élévation de la température du sol, il est nécessaire de connaître le plus exactement possible, dans l'espace et dans le temps, la position des zones de la plante en croissance et/ou en développement par rapport au champ de température. On a donc été amené, pour 4 cultivars représentatifs des 4 espè- ces étudiées, à mesurer systématiquement la taille des principaux organes de la plante et à les resituer par rapport à la surface du sol.

Dans le cas du glaïeul, pour le cultivar « Déciso » (fig. 6), on peut constater que les entrenœuds de la tige, la base des feuilles (zone d'élongation) et l'apex demeurent sous la surface du sol pendant environ les $2 / 3$ de la durée totale de la culture. Pendant toute cette période, ces zones et les processus dont elles sont le siège sont directement influencés par la température du sol.

En ce qui concerne la tulipe et l'iris (fig. 7), si on excepte le degré de différenciation de la fleur qui est plus avancé au moment de la plantation, le mode de croissance est assez semblable à celui du glaïeul.

Par contre, dans le cas du lis (fig. 8), la croissance très rapide du $1^{\text {er }}$ entrencud amène l'apex, les autres entrenouds ainsi que les feuilles sous la dépendance de la température de l'air, ce qui limite l'influence de la température du sol et explique la faible accélération de croissance et de développement que nous avons pu observer pour cette espèce.

\section{EFFETS DU CHAUFFAGE DU SOL SUR LA QUALITÉ DES PLANTES}

Le chauffage du sol se traduit de façon systématique par une diminution du poids des plantes. Elle est particulièrement nette pour le glaïeul (tabl. 6), mais elle affecte également l'iris pour les plantations de novembre à janvier et la tulipe pour celle de décembre. FORTANIER \& ZEVENBERGEN (1973) avaient fait la même observation sur iris et montré que la perte de poids était surtout due à la réduction de la durée de culture engendrée par le chauffage du sol.

Deux phénomènes semblent spécifiques au chauffage du sol :

- la diminution du pourcentage de matière sèche des 


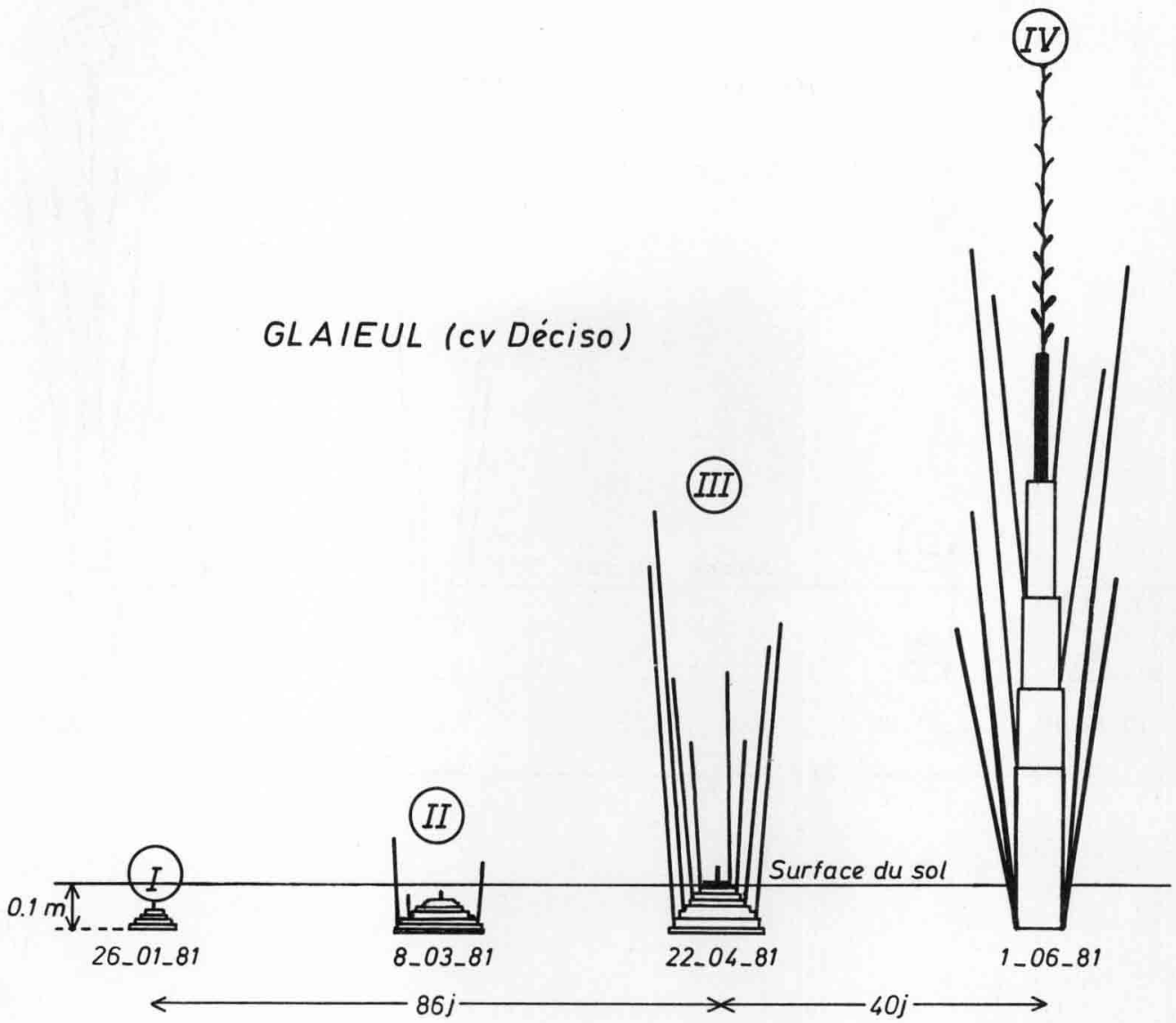

Figure 6

Mode de croissance de la partie aérienne du glaïeul établi à partir de mesures faites sur le cv "Déciso " en sol non chauffé (seules les hauteurs des différents organes sont à l'échelle). Stades repères : I plantation, II levée des feuilles, III levée de la fleur, IV floraison.

Growth of the aerial parts of gladiolus cv. "Deciso " in non-heated soil (only the heights of the different organs are drawn to scale). I planting, II leaf emergence, III flower emergence, IV flowering.

TABLEAU 6

Effet de la réduction de la durée de culture sur la qualité des plantes issues de culture de gläeuls de plein champ menée avec 4 équilibres de température air-sol.

Effect of reduced growth period on the quality of gladiolus on heated soil.

\begin{tabular}{|c|c|c|c|c|}
\hline & $\begin{array}{l}\text { Champ couv. } \\
\text { T sol }=19^{\circ}\end{array}$ & $\begin{array}{c}\text { Champ nu } \\
T \text { sol }=17,4^{\circ}\end{array}$ & $\begin{array}{l}\text { Témoin couv. } \\
\mathrm{T} \text { sol }=15^{\circ}\end{array}$ & $\begin{array}{l}\text { Témoin nu } \\
\mathrm{T} \text { sol }=13^{\circ}\end{array}$ \\
\hline Poids de la plante entière ( $\mathrm{cn}$ g) & 146,41 & 149,2 & 164,78 & $191 ; 13$ \\
\hline Poids du cormus-fils (g) & 14,6 & 16,9 & 18,1 & 19,8 \\
\hline Taille finale de la plante $(\mathrm{cm})$ & 114,8 & 117,4 & 118,0 & 125,9 \\
\hline $\begin{array}{l}\text { Duréc de la phase plantation-floraison } \\
\text { (nombre de jours) }\end{array}$ & 102 & 110 & 118 & 129 \\
\hline
\end{tabular}

\section{TABLEAU 7}

Augmentation (exprimée en \% par rapport au témoin) du poids frais des bulbes fils de la tulipe sur sol chauffé (campagne 1980-1981). Increase in the fresh weight of daughter bulbs on heated soil (19801981).

\begin{tabular}{lllll}
\hline & E. Peeck & Gander & Apcldoorn & K. Nelis \\
\hline$\%$ d'augmentation $+11 \%$ & $+95 \%$ & $+55 \%$ & $+70 \%$ \\
\hline
\end{tabular}

plantes à cause de l'augmentation du taux d'absorption d'eau par les racines,

- le grossissement plus rapide des bulbes fils chez la tulipe (tabl. 7).

$\mathrm{Ce}$ dernier phénomène est particulièrement préoccupant : en effet, si les conditions d'enracinement sont mauvaises, il peut s'accompagner de la réduction de la croissance des plantes et de l'avortement des boutons floraux. On peut rapprocher ces symptômes des observations faites par LE NARD \& COHAT (1982) sur des tulipes dont l'enraci- 


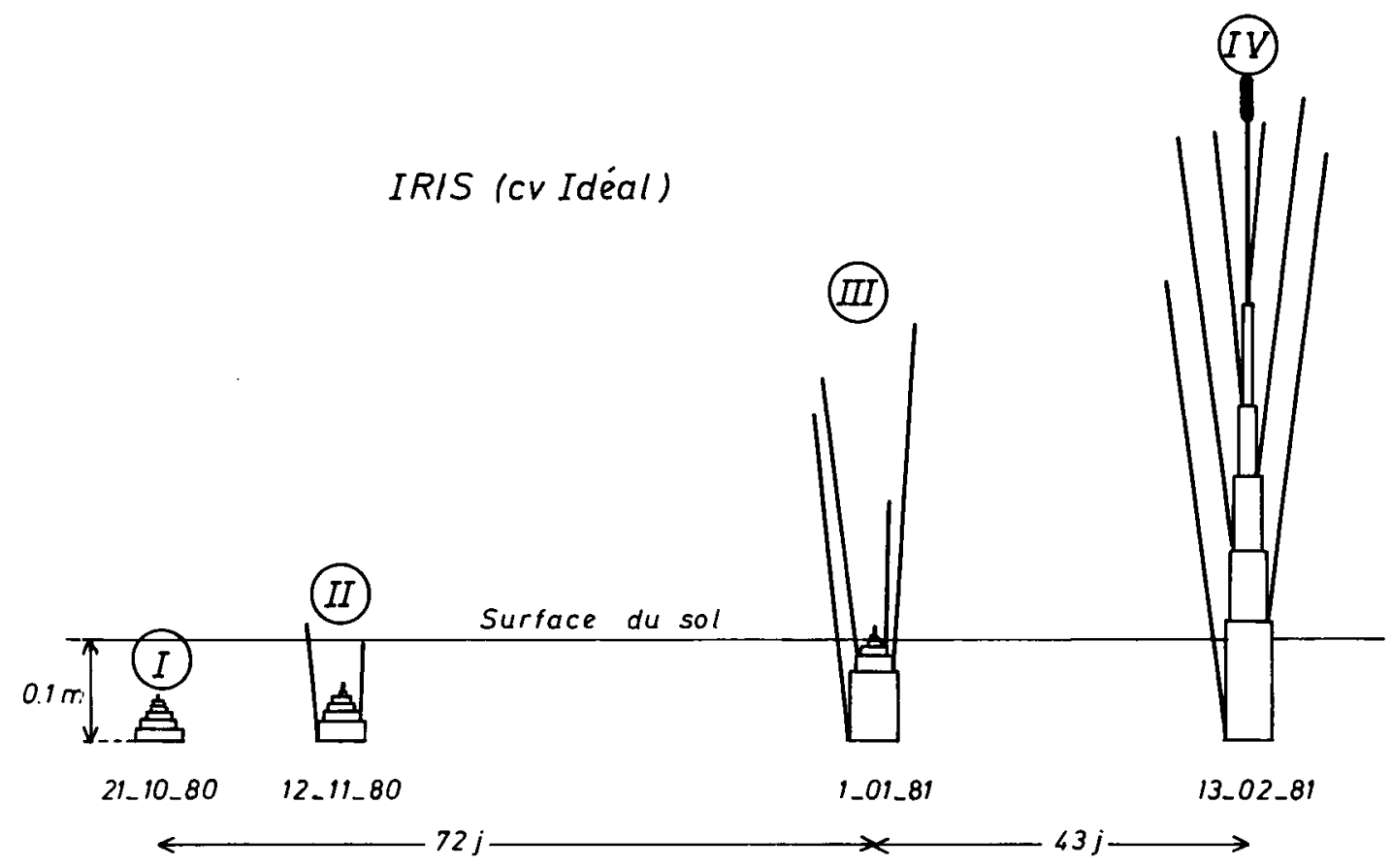

TULIPE (cV Apeldoorn)

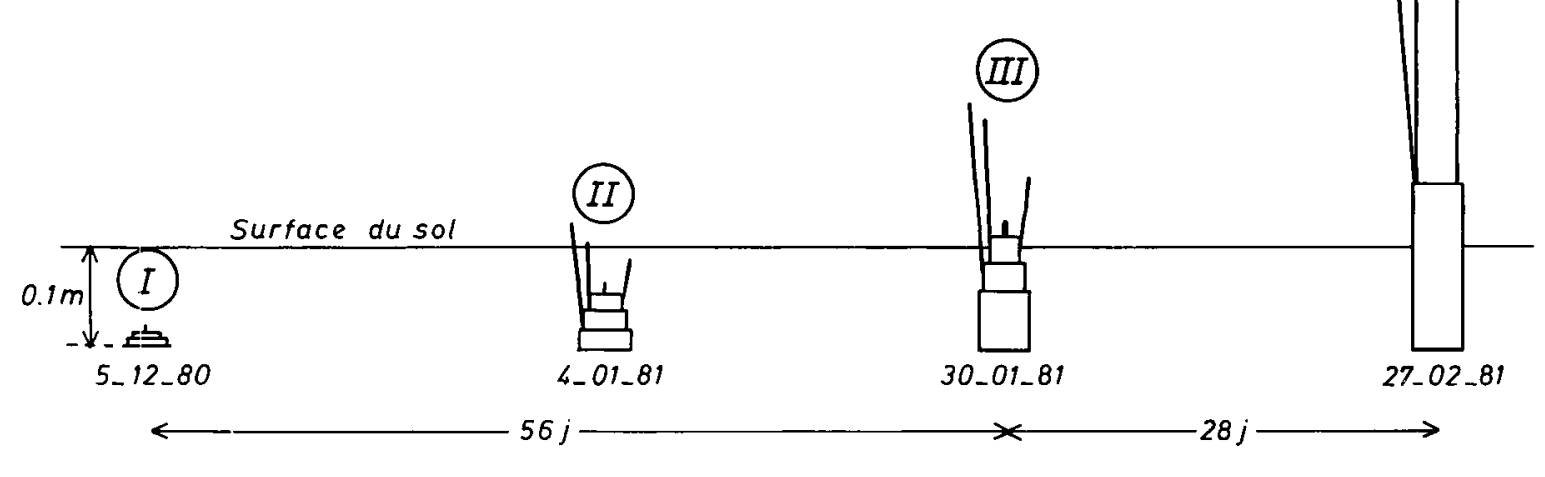

Figure 7

Mode de croissance de la partie aérienne de l'iris et de la tulipe établi à partir de mesures faites sur les cv Idéal et Apeldoorn en sol non chauffé (seules les hauteurs des différents organes sont à l'échelle). Stades repères: I plantation, II levée des feuilles, III sortie de la fleur au-dessus de la surface du sol, IV floraison.

Growth of the aerial parts of iris cv. Ideal and tulip cv. Apeldoorn in non-heated soil (only the heights of the different organs are drawn to scale). I planting, II leaf emergence, III flower emergence, IV flowering.

nement ne peut se réaliser rapidement après la plantation. ce qui les amène à distinguer le processus de bulbification du processus d'élongation et à remarquer : «si la bulbification peut se déclencher la première, l'ćlongation est réduite ou nulle et, dans presque tous les cas, la fleur avorte ».

En général, en observe donc un antagonisme entre la qualité des plantes et le raccourcissement de la duréc de culture.

\section{CONCLUSION}

On peut constater que, dans l'ensemble, les plantes bulbeuses répondent favorablement au chauffage du sol. On a pu mettre en évidence que la température du sol a une influence directe sur leur croissance et leur développement, mais il semble difficile d'analyser séparément ses effets sur 


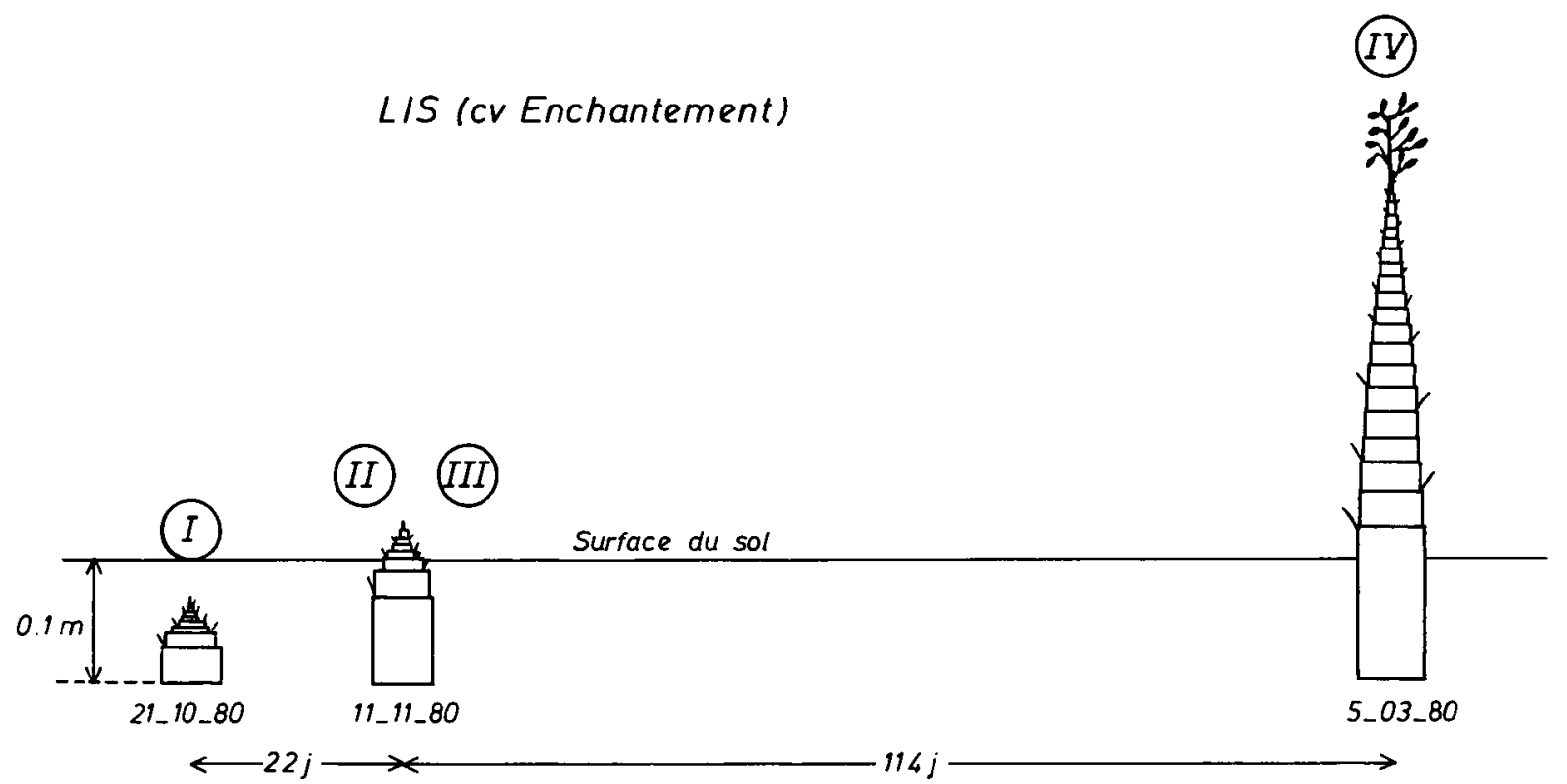

Figure 8

Mode de croissance du lis cv Enchantement établi à partir des mesures faites en sol non chauffé (seules les hauteurs des différents organes sont à l'échelle). I plantation, II levée des feuilles, III sortie de l'apex, IV floraison.

les différents organes de la plante (racine, bulbe, tige, feuilles). Cependant l'antagonisme que l'on constate entre le raccourcissement de la durée de la culture et la qualité des plantes obtenues conduit à déconseiller une température du sol trop élevée.

Au niveau pratique, il semblerait logique de préconiser pour ce type de plantes un chauffage uniquement du sol pendant la période de croissance souterraine, puis un chauffage d'air après l'émergence des fleurs hors du sol.

Cette technique permettrait :

- de raccourcir la durée des cultures, donc la durée de chauffage,

- d'utiliser uniquement un chauffage de sol modéré pendant une grande partic de la culture, ce qui peut entraîner des économies d'énergie substantielles (surtout si l'énergie utilisée est gratuite ou peu onéreusc) du fait des faibles niveaux d'énergie requis $\left(30 \mathrm{~W} \cdot \mathrm{m}^{-2}\right.$ environ) pour maintenir lc sol à la température adéquate (BoulARD \& BAILLE, 1984).
Growth of lily cr. Enchantment in non-heated soil (only the heights of the different organs are drawn to scale). I Planting, II leaf emergence, III apex emergence, IV flowering.

Des essais plus appliqués sont actucllement entrepris pour optimiser la localisation des apports de chalcur, dans l'espace et dans le temps, en fonction du stade de développement des plantes.

Reçu le 14 mars 1983. Accepté le 13 octobre 1983.

\section{REMERCIEMENTS}

Les auteurs tiennent à remercier :

- M. Mevel de l'Institut Technique Interprofessionnel de l'Horticulture, pour les conseils qu'il a apportés dans l'ćlaboration des essais,

- le Centre d'Etude pour l'Utilisation des Rejets thermiques en Région Centre ainsi qu'EDF (Région d'équipement de Tours) pour leur appui technique et financier.

\section{RÉFÉRENCES BIBLIOGRAPHIQUES}

Berninger E., 1977. Essai de cultures florales à différentes températures (Fréjus 1974-1977). Pépiniéristes Hortic. Maraîchers, 181, 2940.

Berninger E., 1978. Mise au point sur le chauffage de l'air et du sol en scrre. Pépiniéristes Hortic. Maraîchers, 191, 13-19.

Blaauw A. H., 1941. On the relation between flower formation and tempcrature (bulbous irises) I ct II. Proc. Kon. Akad. Wet., 44, 513-520 et 684-689.

Boulard T., Baille A., 1984. Utilisation d'eau à basse température pour le forçage de cultures sous abris. I. Aspects microclimatique et énergétique. Agronomie, 4 (3), 213-220.
Boulard T., Baille A., Mevel A., 1981. Chauffage du sol à partir d'eau de rejet sur une culture d'Iris hollandica sous abri : aspects bioclimatologique, thermique, agronomique. Acta Hortic., 115 , 627-633.

Cooper A. J., Thornley A. J., 1976. Response of dry matter partitioning, growth and carbon and nitrogen levels in the tomato plant to changes in root temperature : experiment and theory. Ann. Bot., 40, 1139-1152.

Cornillon P., 1974. Comportement de la tomate en fonction de la température du substrat. Ann. Agron., 25 (5), 753-777. 
Durand R., 1969. Signification et portée des sommes de températurcs. Bull., Tech. Inf. Min. Agric., 238, 185-190.

Durand R., Bonhomme R., Derieux M., 1982. Seuil optimal des sommes de tempćratures. Application au maïs (Zea mays L.). Agronomie, 2 (7), 589-597.

Fortanier E. J., Zevenbergen A., 1979. Analysis of the effects of tempcrature and light after planting on bud blasting in Iris hollandica. Neth. J. Agric. Sci., 21, 145-162.

Hartsema A. M., 1961. Influcnec of temperature on flower formation and flowering of bulbous and tuberous plants. Handbuch der
Pflanzenphysiologie. W. Ruhland (Ed.), 16, 123-167, Soringer Verlag, Berlin.

Le Nard M., 1980. Influence de différents traitements thermiques sur l'intensité de l'induction de la bulbification et sa suppression chez le bulbe de tulipe. Ann. Amelior. Plant., 30 (4), 455-468.

Le Nard M., Cohat J., 1982. Recherches sur la biologie de la tulipe ct du glaïcul. Bull. Tech. Inf. Min. Agric., 366, 3-25.

Schipper J. A., Van Dijk P., 1979. L'emploi du chauffage du sol sur la floraison précoce de l'iris. (D'après des travaux non publićs de Fortanicr). Blcembollencultuur, 21, 494-95. 